\title{
Does the leverage effect affect the return distribution?
}

\author{
Dangxing Chen \\ Consortium for Data Analytics in Risk, Department of Economics, 530 Evans Hall, \\ University of California, Berkeley, CA, 94720-3880, USA
}

\begin{abstract}
The leverage effect refers to the generally negative correlation between the return of an asset and the changes in its volatility. There is broad agreement in the literature that the effect should be present for theoretical reasons, and it has been consistently found in empirical work. However, a few papers have pointed out a puzzle: the return distributions of many assets do not appear to be affected by the leverage effect. We analyze the determinants of the return distribution and find that the impact of the leverage effect comes primarily from an interaction between the leverage effect and the mean-reversion effect. When the leverage effect is large and the mean-reversion effect is small, then the interaction exerts a strong effect on the return distribution. However, if the mean-reversion effect is large, even a large leverage effect has little effect on the return distribution. To better understand the impact of the interaction effect, we propose an indirect method to measure it. We apply our methodology to empirical data and find that the S\&P 500 data exhibits a weak interaction effect, and consequently its returns distribution is little impacted by the leverage effect. Furthermore, the interaction effect is closely related to the size factor: small firms tend to have a strong interaction effect and large firms tend to have a weak interaction effect.
\end{abstract}

Keywords: Leverage effect; Stochastic volatility; Size; Risk.

\footnotetext{
Email address: dangxing@berkeley.edu (Dangxing Chen)

${ }^{1}$ This work was supported by Southwest University of Finance and Economics through the Consortium for Data Analytics in Risk.
}

Preprint submitted to Elsevier 
JEL classification: G11, G12, G17, C58.

\section{Introduction}

The leverage effect refers to the observed tendency of changes in an asset's volatility to be negatively correlated with the asset's returns. The original interpretation goes back to Black (1976). The decline of the stock increases the financial leverage and thereby increases the volatility. Conversely, increases in volatility must be compensated by increases in expected future returns, which can only be achieved by lowering the current stock price. There are many subsequent papers examining the cause of the leverage effect (see, e.g., Christie, 1982; Figlewski and Wang, 2000; French et al., 1987; Campbell and Hentschel, 1992).

Whatever the cause(s) of the leverage effect, there is broad agreement in the literature that the effect is present. Many papers have attempted to accurately estimate the leverage effect (see, e.g., Wang and Mykland, 2014; Bandi and Renò, 2012; Yu, 2005). For example, Ait-Sahalia et al. (2013) calculated that the correlation parameter at the high-frequency limit is $\rho=-0,77$ by high-frequency data of S\&P 500 data with a robust estimation, indicating that there is a strong leverage effect. However, when stochastic volatility models (SVMs) are fitted to the S\&P 500, a puzzle arises: the fitting is insensitive to the correlation parameter (see, e.g., Chorro et al., 2018; Drgulescu and Yakovenko, 2002; Sepp, 2008). How can it be that the return distribution is insensitive to the correlation parameter? This puzzle is the focus of this paper.

Our studies rely on the framework of the continuous-time (CT) SVM. The CT-SVM has been widely successful under both the risk-neutral measure (see, e.g., Sepp, 2008; Heston, 1993; Forde and Jacquier, 2009; Ahn and Gao, 1999; Ait-Sahalia, 2002) and the physical measure (see, e.g., Drgulescu and Yakovenko, 2002; Silva and Yakovenko, 2003; Bakshi et al., 2006). The main advantages are that the CT-SVM is mathematically well-defined and fits the empirical data very well. There are also many useful discrete-time (DT) SVM (see, e.g., 
Bauwens et al., 2006; Duan, 1995; Lamoureux and Lastrapes, 1990). In particular, the DT-SVM EGARCH model derived by Nelson (1991) is widely used in practice. However, the CT-SVM usually has a more flexible form than the DTSVM, and the discretization from continuous time to discrete time doesn't seem always appropriate. For instance, in empirical data, the annualized marginal variance may grow over time; this is not allowed in the EGARCH model. Therefore, in this paper, we would only focus on the CT-SVM.

Under our framework, using the stochastic volatility model of Heston (1993) as an example, we see that the leverage effect is important, but it need not have a strong impact on the return distribution. In particular, the impact of a strong leverage effect on the return distribution can be negated by a strong meanreversion effect. Hence, when studying the distribution properties, one must consider the interaction effect between the leverage effect and the mean-reversion effect. The interaction effect is not directly measurable, since volatility is latent and the Brownian motion is not observable. We propose an indirect method, relying on calculating the dynamics of the marginal variance. A direct impact of this measurement is that the annualized marginal variance will grow over time for a strong interaction effect, but barely change for a weak interaction effect. This phenomenon also provides a better understanding of the performance of the square-root-of-time rule (SRTR) (see, e.g., Danielsson and Zigrand, 2006; Wang et al., 2011; Chen and Anderson, 2018). It is well known that, in the presence of a strong mean-reversion effect, SRTR underpredicts annual volatility when current volatility is low, and overpredicts annual volatility when current volatility is high. We show that, even with a weak mean-reversion effect, if the leverage effect is strong, then the interaction effect has a strong impact on the return distribution. As a result, the SRTR tends to underpredict annual volatility on average.

We apply our methodology to study the relationship between the interaction effect with firm size. The empirical evidence indicates that the interaction effect is strong for small firms but weak for large firms. As a consequence, the annualized marginal variance grows over time for small firms but barely change 
for large firms.

Finally, we aim to explain the leverage effect puzzle in S\&P 500. By using the generalized hyperbolic distribution (see Eberlein et al., 1995), a distribution that arises from the CT-SVM when $\rho=0$, fits the S\&P 500 return distribution very well, despite the fact that the $\mathrm{S} \& \mathrm{P} 500$ exhibits a strong leverage effect. By our method, we show that the interaction effect of the S\&P 500 is weak, answering the puzzle.

The paper is organized as follows. In Section 2, we introduce our basic framework of the stochastic volatility model, followed with a detailed example, the Heston model. Section 3 documents the presence of the leverage effect puzzle, and provides the explanation and the solution. The relationship of the interaction effect with firm size is explored in Section 4. Section 5 studies the leverage effect puzzle in S\&P 500. Section 6 concludes.

\section{Stochastic volatility model}

We assume the price of a security follows the stochastic differential equation $(\mathrm{SDE})$

$$
\begin{aligned}
& d S_{t}=r S_{t} d t+\sqrt{V_{t}} S_{t} d \widetilde{B}_{t}, \\
& d V_{t}=\mu\left(V_{t}\right) d t+\sigma\left(V_{t}\right) d W_{t} .
\end{aligned}
$$

where $\widetilde{B}_{t}$ and $W_{t}$ are two standard Brownian motions with $\mathbb{E}\left[d \widetilde{B}_{t} d W_{t}\right]=\rho d t$, and $r$ is the rate of return 2 Note that

$$
\rho=\lim _{s \rightarrow 0} \operatorname{Corr}\left(V_{t+s}-V_{t}, X_{t+s}-X_{t}\right)
$$

so that the leverage effect is summarized by the correlation parameter $\rho$ under the model (2.1) and (2.2). It is convenient to apply the Gram-Schmidt process to rewrite the price dynamics in terms of two independent Brownian motions

$$
d S_{t}=r S_{t} d t+\rho \sqrt{V_{t}} S_{t} d W_{t}+\sqrt{1-\rho^{2}} \sqrt{V_{t}} S_{t} d B_{t} .
$$

\footnotetext{
${ }^{2}$ Estimates of the mean rate of return are notoriously noisy even over periods of years or decades. Since we cannot make meaningful estimates of $r_{t}$, we might as well assume it as constant.
} 
The $\log$ price dynamics $X_{t}=\ln \left(S_{t}\right)$ can be derived by Itô's Lemma,

$$
d X_{t}=\left(r-\frac{1}{2} V_{t}\right) d t+\rho \sqrt{V_{t}} d W_{t}+\sqrt{1-\rho^{2}} \sqrt{V_{t}} d B_{t} .
$$

Notice that, for greater generality, we don't specify the detailed form of the variance process. The choice of $\mu\left(V_{t}\right)$ and $\sigma\left(V_{t}\right)$ can be quite flexible. We assume that the initial variance $V_{0}>0$ is a realization from the stationary (invariant) distribution of (2.2), so that $V_{t}$ is a stationary process.

We will focus on the marginal return distribution, since it can be directly estimated from the empirical data. We derive some asymptotic properties of the marginal return distribution. We consider the centralized and scaled return distribution

$$
\widetilde{X}_{t}=\frac{X_{t}-\mathbb{E}\left[X_{t}\right]}{\sqrt{t}}
$$

At a short horizon $t \rightarrow 0$, we have

$$
\widetilde{X}_{t} \mid V_{0} \rightarrow \mathcal{N}\left(0, V_{0}\right) .
$$

With this expression, the related moments can be calculated

$$
\begin{aligned}
\operatorname{Var}\left[\widetilde{X}_{t}\right] & =\mathbb{E}\left[V_{0}\right], \\
\text { Skewness }\left[\widetilde{X}_{t}\right] & =0, \\
\text { Kurtosis }\left[\widetilde{X}_{t}\right] & =3+3 \frac{\operatorname{Var}\left[V_{0}\right]}{\mathbb{E}^{2}\left[V_{0}\right]} .
\end{aligned}
$$

Empirically, as one increases the time scale over which returns are calculated, their distributions looks more and more like a Gaussian distribution, as discussed by Cont (2001) . At a long horizon $t \rightarrow \infty$, under some mild conditions (see Peligrad, 1986), which we believe is satisfied empirically, we have

$$
\widetilde{X}_{t} \rightarrow \mathcal{N}\left(0, \frac{\operatorname{Var}\left[X_{t}\right]}{t}\right)
$$

The central moments of the Gaussian distribution are well known 3

$$
\mathbb{E}\left[\widetilde{X}_{t}^{p}\right]=\left\{\begin{array}{l}
0, \quad \text { if } p \text { is odd } \\
\left(\frac{\operatorname{Var}\left[X_{t}\right]}{t}\right)^{p / 2}(p-1) ! !, \quad \text { if } p \text { is even. }
\end{array}\right.
$$

\footnotetext{
${ }^{3}(p-1) !$ ! denotes the double factorial, i.e. the product of all odd numbers $1, \ldots,(p-1)$.
} 


\subsection{Heston model}

Throughout the paper, we will frequently use the stochastic volatility model

of Heston (1993), which has the advantage of providing explicit expressions for many distributional properties. The equation is written as

$$
\begin{aligned}
d X_{t} & =\left(r-\frac{1}{2} V_{t}\right) d t+\rho \sqrt{V_{t}} d W_{t}+\sqrt{1-\rho^{2}} \sqrt{V_{t}} d B_{t}, \\
d V_{t} & =\kappa\left(\theta-V_{t}\right) d t+\sigma \sqrt{V_{t}} d W_{t} .
\end{aligned}
$$

The dynamic of the variance process in the Heston model is also known as the Cox-Ingersoll-Ross (CIR) process (see Cox et al., 2005). We assume in what follows that the Feller condition $2 \kappa \theta>\sigma^{2}$ holds, which guarantees that the variance process is always strictly positive.

The marginal density function can be written in terms of the Fourier integral (see Drgulescu and Yakovenko, 2002)

$$
P_{t}(x)=\frac{1}{2 \pi} \int_{-\infty}^{+\infty} e^{i p_{x} x+F_{t}\left(p_{x}\right)} d p_{x}
$$

with

$$
\begin{aligned}
F_{t}\left(p_{x}\right) & =\frac{\kappa \theta}{\sigma^{2}} \Gamma t-\frac{2 \kappa \theta}{\sigma^{2}} \ln \left[\cosh \left(\frac{\Omega t}{2}\right)+\frac{\Omega^{2}-\Gamma^{2}+2 \kappa \Gamma}{2 \kappa \Gamma} \sinh \left(\frac{\Omega t}{2}\right)\right], \\
\Gamma & =\kappa+i \rho \sigma p_{x} \\
\Omega & =\sqrt{\Gamma^{2}+\sigma^{2}\left(p_{x}^{2}-i p_{x}\right)} .
\end{aligned}
$$

With this, the marginal density function of return can be recovered via the fast Fourier transform (see, e.g., Valsa and Brančik, 1998; Abate and Whitt, 1995)

Many formulas regarding the marginal moments can then be derived from Formula (2.14). For an intuitive understanding, we consider the first four moments. The marginal expectation is given by

$$
\mathbb{E}\left[X_{t}\right]=r t-\frac{\theta t}{2} .
$$

The marginal variance is given by

$\operatorname{Var}\left[X_{t}\right]=\mathbb{E}\left[\int_{0}^{t} V_{s} d s\right]+\frac{1}{4} \operatorname{Var}\left[\int_{0}^{t} V_{s} d s\right]-\rho \mathbb{E}\left[\left(\int_{0}^{t} V_{s} d s\right)\left(\int_{0}^{t} \sqrt{V_{s}} d W_{s}\right)\right]$. 
and the expression for these terms are

$$
\begin{aligned}
\mathbb{E}\left[\int_{0}^{t} V_{s} d s\right] & =\theta t, \\
\mathbb{E}\left[\left(\int_{0}^{t} V_{s} d s\right)\left(\int_{0}^{t} \sqrt{V_{s}} d W_{s}\right)\right] & =\theta \frac{\sigma}{\kappa}\left[t+\frac{e^{-\kappa t}-1}{\kappa}\right], \\
\operatorname{Var}\left[\int_{0}^{t} V_{s} d s\right] & =\theta \frac{\sigma^{2}}{\kappa^{2}}\left[t+\frac{e^{-\kappa t}-1}{\kappa}\right] .
\end{aligned}
$$

The marginal skewness is

$$
\text { Skewness }\left[X_{t}\right]=\frac{\mathbb{E}\left[\left(X_{t}-\mathbb{E}\left[X_{t}\right]\right)^{3}\right]}{\operatorname{Var}\left[X_{t}\right]^{3 / 2}},
$$

where

$$
\begin{aligned}
\mathbb{E}\left[\left(X_{t}-\mathbb{E}\left[X_{t}\right]\right)^{3}\right] & =\frac{3}{8 \kappa^{5}} e^{-\kappa t} \theta \sigma(-2 \kappa \rho+\sigma)\left(-4 \kappa^{2}+8 \kappa \rho \sigma+4 t \kappa^{2} \rho \sigma-2 \sigma^{2}-t \kappa \sigma^{2}\right. \\
& \left.+e^{\kappa t}\left(-4 \kappa^{2}(-1+t \kappa)+4 \kappa(-2+t \kappa) \rho \sigma+(2-t \kappa) \sigma^{2}\right)\right)
\end{aligned}
$$

The marginal kurtosis is

$$
\operatorname{Kurtosis}\left[X_{t}\right]=\frac{\mathbb{E}\left[\left(X_{t}-\mathbb{E}\left[X_{t}\right]\right)^{4}\right]}{\operatorname{Var}\left[X_{t}\right]^{2}}
$$

where

$$
\begin{aligned}
\mathbb{E}\left[\left(X_{t}-\mathbb{E}\left[X_{t}\right]\right)^{4}\right] & =\frac{3}{32 \kappa^{7}} e^{-2 \kappa t} \theta\left(\sigma^{2}(-4 \kappa \rho+\sigma)^{2}\left(2 \theta \kappa+\sigma^{2}\right)\right. \\
& +4 e^{\kappa t} \sigma\left(-16 t \theta \kappa^{5} \rho+4 \kappa^{3}\left((2+t \theta) \kappa+4(\theta(-1+t \kappa)+2 \kappa(2+t \kappa)) \rho^{2}\right) \sigma\right. \\
& -8 \kappa^{2} \rho\left(\theta(-1+t \kappa)+6 \kappa(2+t \kappa)+2 \kappa(6+t \kappa(4+t \kappa)) \rho^{2}\right) \sigma^{2} \\
& +\kappa\left(\theta(-1+t \kappa)+4 \kappa\left(6+3 t \kappa+(34+t \kappa(24+5 t \kappa)) \rho^{2}\right)\right) \sigma^{3} \\
& \left.-8 \kappa(7+t \kappa(5+t \kappa)) \rho \sigma^{4}+(7+t \kappa(5+t \kappa)) \sigma^{5}\right) \\
& +e^{2 \kappa t}\left(2 t^{2} \theta \kappa^{3}\left(4 \kappa^{2}-4 \kappa \rho \sigma+\sigma^{2}\right)^{2}\right. \\
& +2 t \kappa \sigma\left(4 \kappa^{2}-4 \kappa \rho \sigma+\sigma^{2}\right)\left(8 \theta \kappa^{2} \rho+2 \kappa\left(-\theta+2 \kappa+8 \kappa \rho^{2}\right) \sigma-20 \kappa \rho \sigma^{2}+5 \sigma^{3}\right) \\
& +\sigma^{2}\left(-32 \kappa^{4}\left(1+8 \rho^{2}\right)-29 \sigma^{4}+2 \kappa \sigma^{2}(\theta+116 \rho \sigma)+32 \kappa^{3} \rho\left(\theta \rho+12\left(1+\rho^{2}\right) \sigma\right)\right. \\
& \left.\left.\left.-16 \kappa^{2} \sigma(6 \sigma+\rho(\theta+35 \rho \sigma))\right)\right)\right) .
\end{aligned}
$$




\section{The interaction effect}

\subsection{The leverage effect puzzle}

To motivate the analysis that follows, we start with a straightforward artificial example to illustrate the leverage effect puzzle. Figure 1 compares the marginal densities of the return in the Heston model (2.14) with and without the leverage effect for two sets of Heston parameters $\kappa, \sigma$. In the first example with $\kappa=16$ and $\sigma=0.8$, we see a strong impact of the leverage effect. In fact, with $\rho=-1$, the marginal density is quite negatively skewed. In the second example, with $\kappa=1$ and $\sigma=0.02$, we see that the two marginal densities with $\rho=0$ and $\rho=-1$ are almost identical. So why does the impact of the leverage effect almost disappear in some cases? That is the "leverage effect puzzle" that we seek to understand. The goal of this paper is to understand the sources of the puzzle and propose a solution.
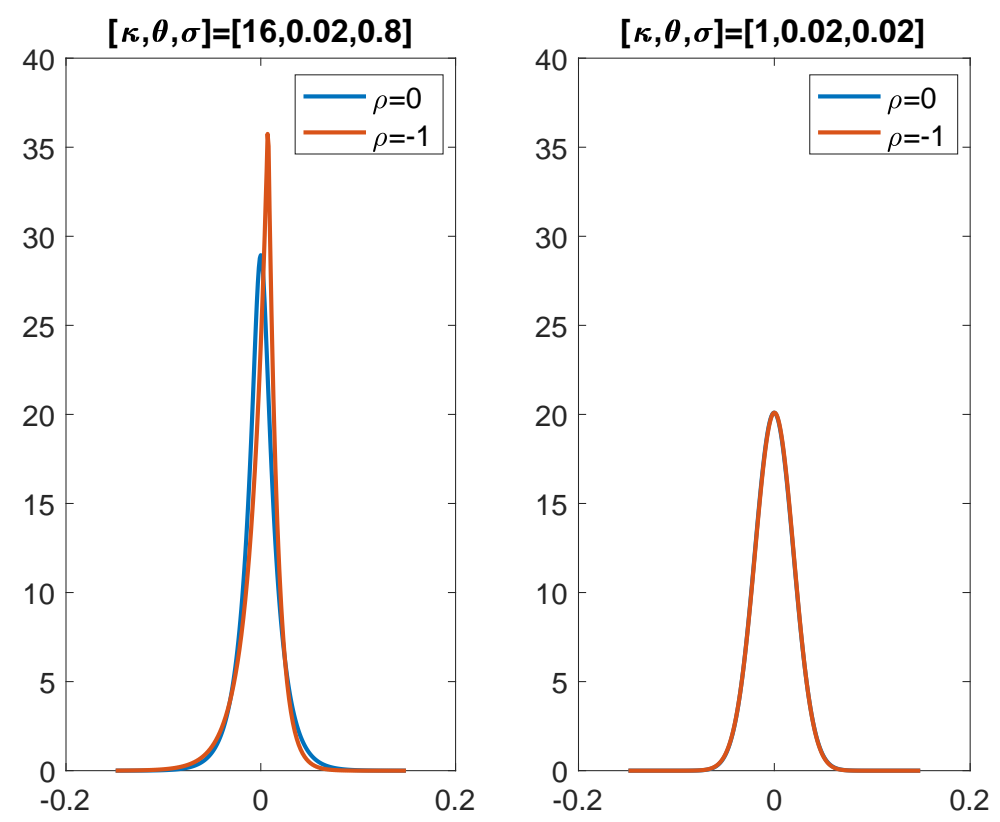

Figure 1: The marginal density of the Heston model at 5-day horizon with and without the leverage effect 


\subsection{Interaction effect}

When the leverage effect is not present, i.e., $\rho=0$, the solution to the SDE (2.5) can be written as

$$
X_{t} \mid \int_{0}^{t} V_{s} d s=\mathcal{N}\left(r-\frac{1}{2} \int_{0}^{t} V_{s} d s, \int_{0}^{t} V_{s} d s\right) .
$$

Now consider another case when there is a strong mean-reversion effect such that $V_{t}$ is independent of $W_{t}$, then the equation (3.1) also holds and the impact of the leverage effect is not observed. This situation could occurs, for example, when the effect from the diffusion term is relatively weak. Returning to the example of the Heston model in Figure 1, let's see the impact of the leverage effect on the marginal moments of return. At a short horizon $t \sim 0$, the following simple approximations follow from equations (2.20), (2.21), and (2.22)

$$
\begin{aligned}
\mathbb{E}\left[X_{t}\right] & \sim r t-\frac{\theta t}{2}, \\
\operatorname{Var}\left[X_{t}\right] & \sim \theta t \\
\operatorname{Skewness}\left[X_{t}\right] & \sim \sigma\left(\frac{3}{2} \rho-\frac{3}{4} \frac{\sigma}{\kappa}\right) \sqrt{\frac{t}{\theta}} \\
\operatorname{Kurtosis}\left[X_{t}\right] & \sim 3+\frac{3 \sigma^{2}}{2 \kappa \theta} .
\end{aligned}
$$

Note the expectation, variance, and kurtosis are not affected by the leverage effect, but the skewness is. Even with a strong leverage effect, if $\sigma$ is small (i.e. the variance process $V_{t}$ is not very volatile), the skewness will be close to 0 and the impact of the leverage effect will be hard to observe.

Hence, a strong leverage effect can be negated by a strong mean-reversion effect. When studying return distributions, it is essential to incorporate the interaction effect, namely the interaction between the mean-reversion and leverage effects. Since the variance is latent and the Brownian motion is not observable, we propose an indirect method to measure the interaction effect.

\subsection{Measurement of the interaction effect}

We need an accurate, robust, and interpretable measure of the interaction effect. Commonly used measurements in summary statistics include the location 
(e.g., mean, median), dispersion (e.g., standard deviation), and shape (skewness and kurtosis). For these measurements, calculations typically rely on moments or quantiles. It would be natural to focus on the moment-based calculations, since these can be readily interpreted in terms of the SDE. Unfortunately, the estimation of moments in financial data may be inaccurate. For example, consider the expectation of return $\mathbb{E}\left[X_{t}\right]=r t-\frac{\theta t}{2}$. At a short horizon, the standard deviation of the return $\sqrt{\operatorname{Var}\left[X_{t}\right]} \sim \sqrt{\theta t}$ has a much larger magnitude than its expectation. As a consequence, we cannot accurately estimate the mean return even with about 100 years of daily return, due to the slow convergence under the central limit theorem. The lack of robustness in the calculation of skewness and kurtosis in financial data using moments is documented in Kim and White (2004) and Bonato (2011).

Therefore, among these common measurements, we focus on the calculation of the marginal variance since it can be estimated accurately and is easily interpretable. Recall that by algebra, the marginal variance can be decomposed into three terms:

$\operatorname{Var}\left[X_{t}\right]=\mathbb{E}\left[\int_{0}^{t} V_{s} d s\right]+\frac{1}{4} \operatorname{Var}\left[\int_{0}^{t} V_{s} d s\right]-\rho \mathbb{E}\left[\left(\int_{0}^{t} V_{s} d s\right)\left(\int_{0}^{t} \sqrt{V_{s}} d W_{s}\right)\right]$.

For simplicity, we use the following notation for these terms:

$$
\begin{aligned}
\operatorname{EIV}_{t} & =\mathbb{E}\left[\int_{0}^{t} V_{s} d s\right] \\
\operatorname{VIV}_{t} & =\frac{1}{4} \operatorname{Var}\left[\int_{0}^{t} V_{s} d s\right], \\
\operatorname{EMIV}_{t} & =-\rho \mathbb{E}\left[\left(\int_{0}^{t} V_{s} d s\right)\left(\int_{0}^{t} \sqrt{V_{s}} d W_{s}\right)\right] .
\end{aligned}
$$

Note that the $\mathrm{EIV}_{t}$ is scalable with respect to time, i.e., $\mathrm{EIV}_{t}=\frac{t}{s} \mathrm{EIV}_{s}$. Due to the mean-reversion effect, empirically, we believe the $\mathrm{VIV}_{t}$ is negligible comparing to the $\mathrm{EIV}_{t}$, i.e., $\mathrm{VIV}_{t} \ll \mathrm{EIV}_{t}$. Then the $\mathrm{EMIV}_{t}$ comes from the contribution from the interaction effect and is the term we want to measure. The EMIV $t$ should be close to 0 for a weak interaction effect, far away from 0 for a strong interaction effect. 
For a more intuitive understanding of these formulas, we use the Heston model as an example, taking formulas from Equations (2.20), (2.21), and (2.22). At a short horizon $t \sim 0$, we have

$$
\begin{aligned}
\mathbb{E}\left[\int_{0}^{t} V_{s} d s\right] & \sim \theta t, \\
\mathbb{E}\left[\left(\int_{0}^{t} V_{s} d s\right)\left(\int_{0}^{t} \sqrt{V_{s}} d W_{s}\right)\right] & \sim \theta \frac{\sigma}{2} t^{2}, \\
\operatorname{Var}\left[\int_{0}^{t} V_{s} d s\right] & \sim \theta \frac{\sigma^{2}}{2 \kappa} t^{2} .
\end{aligned}
$$

Thus, at a short horizon $t \sim 0$, we have $\operatorname{Var}\left[X_{t}\right] \sim \operatorname{EIV}_{t}$. At a long horizon $t \sim \infty$, we have

$$
\begin{aligned}
\mathbb{E}\left[\int_{0}^{t} V_{s} d s\right] & \sim \theta t \\
\mathbb{E}\left[\left(\int_{0}^{t} V_{s} d s\right)\left(\int_{0}^{t} \sqrt{V_{s}} d W_{s}\right)\right] & \sim \theta t \frac{\sigma}{\kappa}, \\
\operatorname{Var}\left[\int_{0}^{t} V_{s} d s\right] & \sim \theta t \frac{\sigma^{2}}{\kappa^{2}} .
\end{aligned}
$$

Thus, the contribution from the $\mathrm{EMIV}_{t}$ can be observed. In practice, the calculation is done by $\operatorname{EMIV}_{t} \approx \operatorname{Var}\left[X_{t}\right]-\frac{t}{s} \operatorname{Var}\left[X_{s}\right]$ for $t>s$. Note that each term is scaled by a factor $\frac{\sigma}{\kappa}$. This term serves as a mean-reversion factor in the Heston model.

From the calculation of the interaction effect by $\operatorname{EMIV}_{t}$, there is a direct impact: the annualized marginal variance will grow over time until it converges when the interaction effect is strong, but it will barely change when the interaction effect is weak. This phenomenon suggests that in practice, if one observes two securities with the same average variance at a short horizon, it doesn't imply that these two securities have the same volatility. If the interaction effect is strong for one and weak for another one, the security with the strong interaction effect will become more volatile over time.

Another advantage of measuring the marginal variance is we know the centralized and scaled return $\widetilde{X}_{t}$ would eventually converge to Gaussian (2.11). Suppose the information of marginal variance is known, we then know the 
asymptotic distribution of the return. In practice, the asymptotic distribution of the return can be easily obtained for a security with a weak interaction effect.

\subsection{Impact on the square-root of time rule}

It is common to use the SRTR (see Danielsson and Zigrand, 2006) to extrapolate the conditional variance of the log return $\operatorname{Var}\left[X_{t} \mid V_{0}\right]$. Under the SVM, the SRTR serves as the constant approximation to the conditional variance

$$
\operatorname{Var}\left[X_{t} \mid V_{0}\right] \approx V_{0} t
$$

We discuss the impact of the mean-reversion effect and the leverage effect on the performance of the SRTR, along with the Heston model as an example in Figure 2. It is well known that, because it ignores the mean-reversion effect, the SRTR tends to under-predict for small initial variance but over-predict for large initial variance. As shown in the first example in Figure 2 due to the meanreversion effect, the conditional variance is flatter than the SRTR. But this is not the whole story; one must also take the interaction effect into account. If the mean-reversion effect is weak and the leverage effect is strong, the resulting strong interaction effect leads SRTR tends to under-predict on average, as shown in the second example of Figure 2. This also explains the downward-biased prediction using the SRTR observed by Wang et al. (2011). In the case of a weak mean-reversion effect and a weak leverage effect, the SRTR serves as a good approximation, as plotted in the third example in Figure 2, 

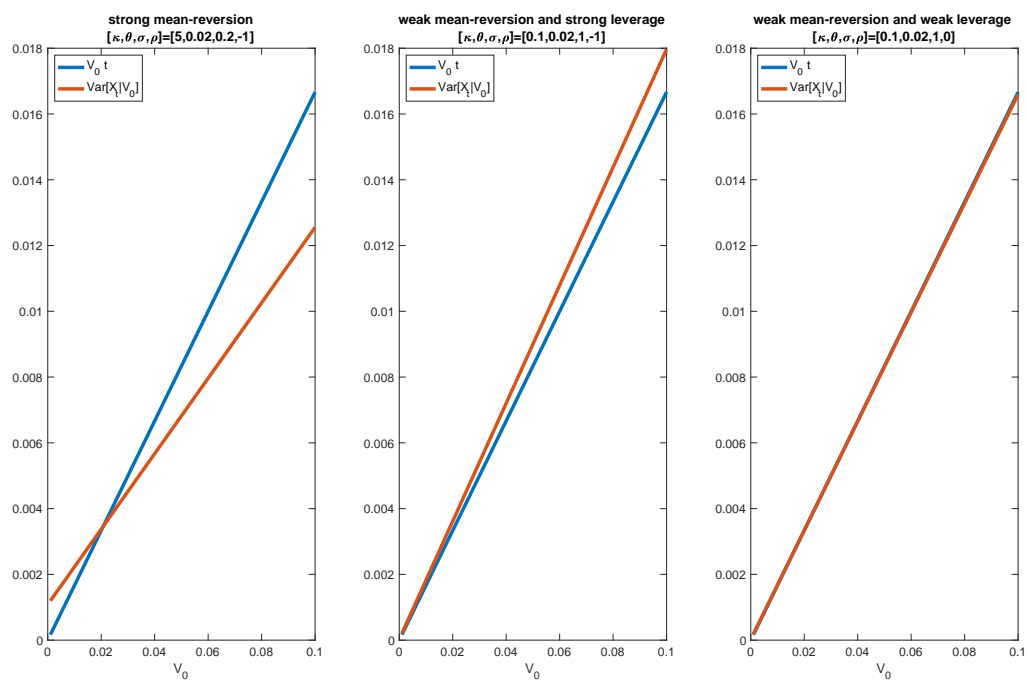

Figure 2: Performance of the square-root-of-time rule by Heston model for different cases at 2-month horizon

\section{Relationship of the interaction effect with firm size}

Here, we use five value-weighted size portfolios constructed by Fama and French 4 , sorted by market capitalization, price times shares outstanding. All NYSE, AMEX, and NASDAQ stocks are included. We use daily data from July 1926 to January 2019.

To study the interaction effect, we compare the dynamics of the marginal variance to the $\operatorname{EIV}_{t}$ over time in Figure 3 From the figure, we observe a clear trend. The gap between the marginal variance at time $t$ and $\operatorname{EIV}_{t}$ grows over time for small firms but is nearly zero for large firms. This implies that the interaction effect is stronger for small firms than larger firms.

\footnotetext{
${ }^{4}$ The data is downloaded from the website http://mba.tuck.dartmouth.edu/pages/faculty/ken.french/data_library.html
} 

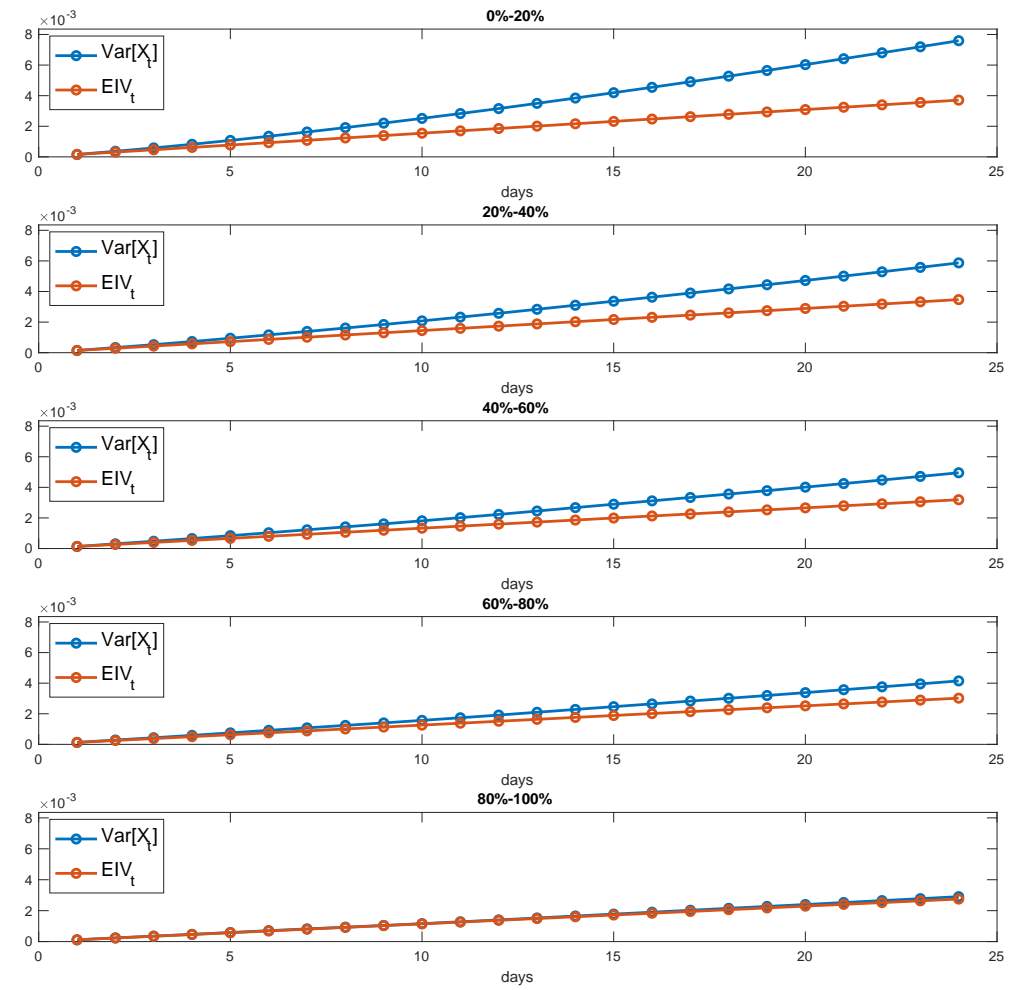

Figure 3: The interaction effect for portfolios constructed by size

For a more quantitative understanding, we also calculate the summary statistics. Define $\mathrm{RIV}_{t}$ as the proportion of the $\mathrm{EMIV}_{t}$ to the marginal variance $\operatorname{Var}\left[X_{t}\right]$,

$$
\operatorname{RIV}_{t}=\frac{\operatorname{Var}\left[X_{t}\right]-\operatorname{EIV}_{t}}{\operatorname{Var}\left[X_{t}\right]}
$$

The summary statistics at the 25-day time horizon are given in Table 1 From the first column, it is observed that the $\mathrm{EIV}_{t}$ is larger for small firms than large firms, implying that at a short horizon, small firms are more volatile than large firms, which is not surprising. From the second column, we see that the interaction effect is stronger for small firms than large firms. In particular, the 
annualized marginal variance roughly doubles for the smallest firms $\left(\operatorname{Var}\left[X_{t}\right]=\right.$ $\left.(1-0.51)^{-1} \mathrm{EIV}_{t}\right)$, but barely changes for largest firms at the 25-day horizon.

Table 1: Summary statistics of the interaction effect for the portfolios constructed by size

\begin{tabular}{ccc}
\hline Portfolios & $\mathrm{EIV}_{25}$ & $\mathrm{RIV}_{25}$ \\
\hline $0 \%-20 \%$ & 0.037 & 0.51 \\
\hline $20 \%-40 \%$ & 0.035 & 0.41 \\
\hline $40 \%-60 \%$ & 0.032 & 0.36 \\
\hline $60 \%-80 \%$ & 0.030 & 0.27 \\
\hline $80 \%-100 \%$ & 0.028 & 0.053 \\
\hline
\end{tabular}

We also check the mean-reversion effect separately for size portfolios. Recall at a short horizon, from (2.10), the excess kurtosis by moments is $3 \frac{\operatorname{Var}\left[V_{0}\right]}{\mathbb{E}^{2}\left[V_{0}\right]}$. Hence, at a short horizon, the excess kurtosis measures the volatility of the variance process $V_{t}$. The excess kurtosis is closely related to the mean-reversion effect: if the variance process $V_{t}$ is not volatile or the mean-reversion effect is strong, the excess kurtosis will be small.

Unfortunately, as pointed out by Kim and White (2004) and Bonato (2011), the measurement of kurtosis by moments is not robust for stock returns. As an alternative, we use the quantile-based measurement developed in Crow and Siddiqui (1967). The centered coefficient is

$$
\text { Excess Kurtosis }_{C S}=\frac{F^{-1}(0.975)-F^{-1}(0.025)}{F^{-1}(0.75)-F^{-1}(0.25)}-2.91,
$$

where $F$ is the empirical cumulative distribution function. Since we have approximately 24,000 daily observations, the 0.025 and 0.975 quantiles can be measured with reasonable accuracy. This formula is applied to size portfolios in Table 2 The results indicate that for small firms, in addition to the price dynamics, the variance process $V_{t}$ is also more volatile than for large firms. This also implies that the mean-reversion effect is weaker for small firms than for large firms and therefore helps to explain why the interaction effect for is stronger for small firms than for large firms. 
Table 2: Summary statistics of the marginal variance, skewness, and kurtosis for the portfolios constructed by size at 1-day horizon

\begin{tabular}{cccc}
\hline Portfolios & Annualized marginal variance & Skewness $_{H}$ & Excess Kurtosis $_{C S}$ \\
\hline $0 \%-20 \%$ & 0.039 & -0.12 & 2.22 \\
\hline $20 \%-40 \%$ & 0.036 & -0.11 & 2.04 \\
\hline $40 \%-60 \%$ & 0.034 & -0.11 & 1.96 \\
\hline $60 \%-80 \%$ & 0.032 & -0.093 & 1.89 \\
\hline $80 \%-100 \%$ & 0.029 & -0.053 & 1.73 \\
\hline
\end{tabular}

We also calculate the skewness using quantiles. We use the measurement by Hinkley (1975)

$$
\text { Skewness }_{H}=\frac{F^{-1}(1-\alpha)+F^{-1}(\alpha)-2 F^{-1}(0.5)}{F^{-1}(1-\alpha)-F^{-1}(\alpha)}
$$

with the choice of $\alpha=0.05$. The result is given in Table 2 . The result indicates that the return distribution is more negatively skewed for small firms than large firms.

\section{Disentangling the puzzle for S\&P 500}

In this section, we explain the observed puzzle that the fitting of the S\&P 500 is insensitive to the correlation parameter $\rho$, even though the empirical data indicates that there is a strong leverage effect $\rho \sim-0.77$.

First, we want to confirm that the impact on the return distribution is indeed weak. To do so, we conduct an experiment. Recall that if there is no leverage effect, the marginal density follows the mixture Gaussian distribution (3.1). We show that we can fit a stochastic volatility model with no leverage effect well to the empirical data. We will rely on the generalized hyperbolic (GH) distribution (see, e.g., Eberlein et al., 1995; BenSaïda and Slim, 2016; Barndorff-Nielsen and Halgreen, 1977; Barndorff-Nielsen, 1977), which has proved to be very powerful empirically. If $Y$ follows a generalized hyperbolic distribution we write

$$
Y \sim H(\lambda, \alpha, \beta, \delta, \mu)
$$


The PDF of a GH distribution is given by

$$
p_{Y}(x)=\frac{(\gamma / \delta)^{\lambda}}{\sqrt{2 \pi} K_{\lambda}(\delta \gamma)} \frac{K_{\lambda-\frac{1}{2}}\left(\alpha \sqrt{\delta^{2}+(x-\mu)^{2}}\right)}{\left(\sqrt{\delta^{2}+(x-\mu)^{2}} / \alpha\right)^{\beta-\lambda}} e^{\frac{1}{2}(x-\mu)},
$$

where $\gamma^{2}=\alpha^{2}-\beta^{2}$, and $K_{\lambda}$ is the modified Bessel function of the third kind with index $\lambda$. The parameter domain for the class of GH distributions is given by

$$
\begin{aligned}
& \delta \geq 0, \alpha>0, \alpha^{2}>\beta^{2}, \text { if } \lambda>0, \\
& \delta>0, \alpha>0, \alpha^{2}>\beta^{2}, \text { if } \lambda=0, \\
& \delta>0, \alpha \geq 0, \alpha^{2} \geq \beta^{2}, \text { if } \lambda<0 .
\end{aligned}
$$

The generalized inverse Gaussian (GIG) distribution (see Seshadri, 2004) is closely related to GH. If $V$ follows a GIG distribution we write

$$
V \sim G I G(\lambda, \delta, \gamma)
$$

The PDF of a GIG distribution is given by

$$
p_{V}(x)=\frac{(\gamma / \delta)^{\lambda}}{2 K_{\lambda}(\delta \gamma)} x^{\lambda-1} e^{-\frac{1}{2}\left(\delta^{2} x^{-1}+\gamma^{2} x\right)}, x>0 .
$$

The parameter domain for the class of GIG distributions is given by

$$
\begin{aligned}
& \delta>0, \gamma \geq 0, \text { if } \lambda<0, \\
& \delta>0, \gamma>0, \text { if } \lambda=0, \\
& \delta \geq 0, \gamma>0, \text { if } \lambda>0 .
\end{aligned}
$$

The GH distribution was originally derived in Barndorff-Nielsen (1977); it is a Gaussian variance-mean mixture where the mixing distribution is GIG. In other words, if

$$
Y \mid V=v \sim \mathcal{N}(\mu+\beta v, v)
$$

and $V \sim \operatorname{GIG}(\lambda, \delta, \lambda)$, then the marginal distribution of $Y$ will be GH, $Y \sim$ $H(\lambda, \alpha, \beta, \delta, \mu)$, where $\alpha^{2}=\beta^{2}+\gamma^{2}$. To connect the GIG to the SDE, Sørensen (1997) considers the SDE

$$
d V_{t}=\left(\beta_{1} V_{t}^{2 \alpha-1}-\beta_{2} V_{t}^{2 \alpha}+\beta_{3} V_{t}^{2(\alpha-1)}\right) d t+\kappa V_{t}^{\alpha} d W_{t}
$$


where $\beta_{1}=\frac{1}{2} \kappa^{2}(\lambda-1)+\kappa^{2} \alpha, \quad \beta_{2}=\frac{1}{4}(\kappa \gamma)^{2}, \quad \beta_{3}=\frac{1}{4}(\kappa \delta)^{2}$. This SDE has a stationary distribution which is GIG. Note that if $\alpha=\frac{1}{2}$, then the diffusion process is the solution to

$$
d V_{t}=\left(\beta_{1}-\beta_{2} V_{t}+\frac{\beta_{3}}{V_{t}}\right) d t+\kappa \sqrt{V_{t}} d W_{t}
$$

which is the CIR-process with an additional $\frac{\beta_{3}}{V_{t}}$ in the drift term. Empirically, the volatility of the market is never zero. In the CIR-process, the variance can become zero if the Feller condition is violated, and imposing the Feller condition limits our ability to calibrate the model to data. The additional $\frac{\beta_{3}}{V_{t}}$ term prevents the variance from becoming zero, while leaving more freedom to fit the data.

The fitting of the empirical distribution of the S\&P 500 by GH is shown in Figure 4. Visually, the fit is virtually perfect. Quantitatively, we apply the Kolmorogov-Smirnov test (see, e.g., Weiss, 1978; Chicheportiche and Bouchaud, 2011) to verify with daily returns drawn one-month apart so that the data is only very weakly dependent. The test fails to reject the null hypothesis that the sample is drawn from the $\mathrm{GH}$ distribution at the $5 \%$ significance level, confirming that we have a good fit. This experiment, which shows that it is possible to fit the S\&P 500 return distribution with the GH distribution, which is derived under the assumption of zero leverage effect, confirms that the leverage effect has only a very weak impact on the S\&P 500 return distribution. 


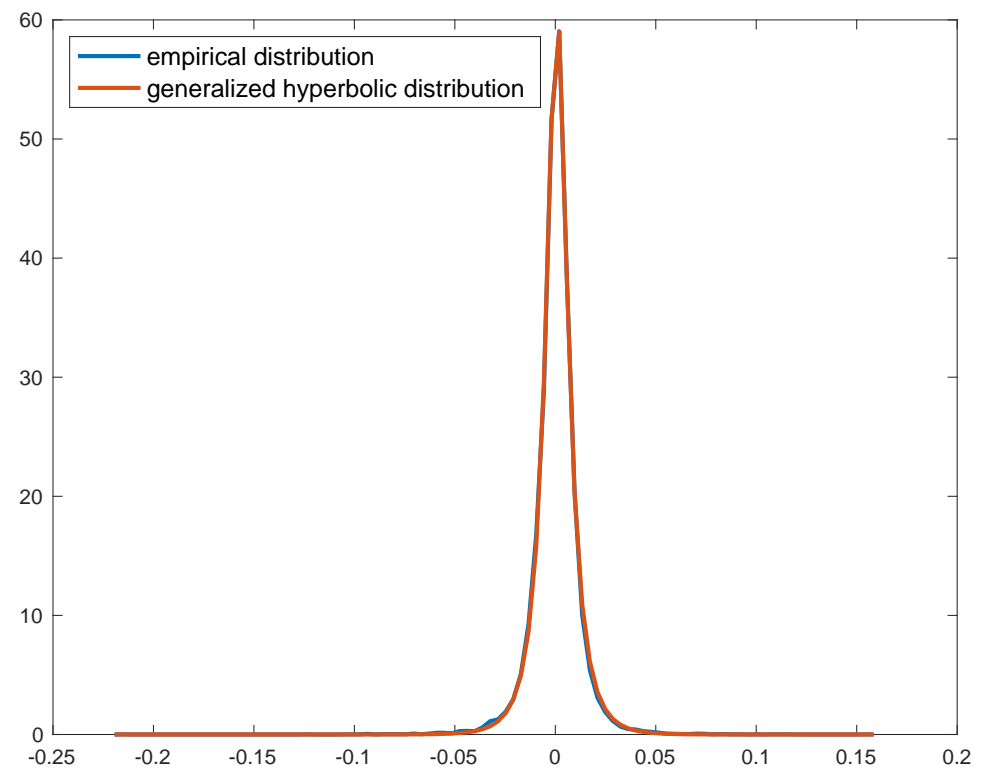

Figure 4: The fitness of the S\&P 500 by the generalized hyperbolic distribution at the daily scale 


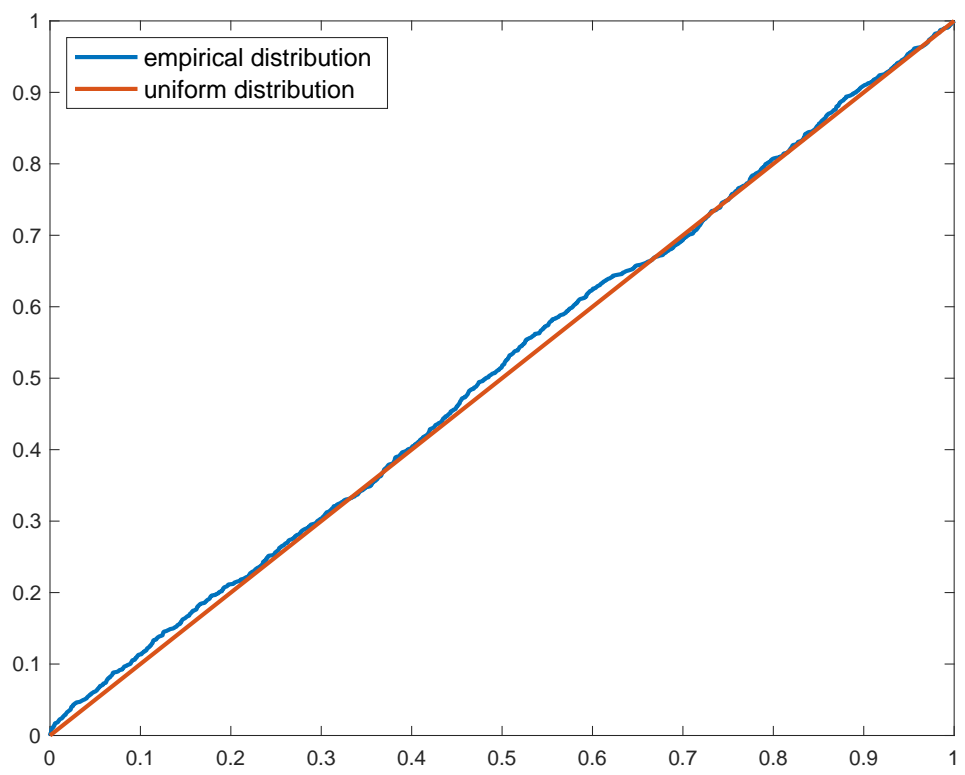

Figure 5: The Kolmorogov-Smirnov test test of the S\&P 500 at the daily scale

We now turn to the interaction effect for the S\&P 500. The comparison of the dynamics of marginal variances with $\operatorname{EIV}_{t}$ is plotted in Figure 6. The marginal variance is quite close to $\mathrm{EIV}_{t}$, indicating that the interaction effect is quite weak. We believe this weak interaction effect is the reason that the leverage effect has little impact on the distribution for S\&P 500.

To back up our story, we check the mean-reversion property of the S\&P 500. We found that the interaction effect is weak for the S\&P 500. From the literature, the leverage effect is strong. By our analysis, this implies that the mean-reversion effect for S\&P 500 must be strong. The excess kurtosis, calculated using Equation (4.1) of S\&P 500 is relatively low, at 1.72, which confirms that the mean-reversion effect is strong, consistent with our analysis 5

\footnotetext{
${ }^{5}$ The result is quite similar to that for large firms, 1.73. This is not surprising since the $\mathrm{S} \& \mathrm{P} 500$ is composed essentially of the 500 largest firms by market capitalization.
} 


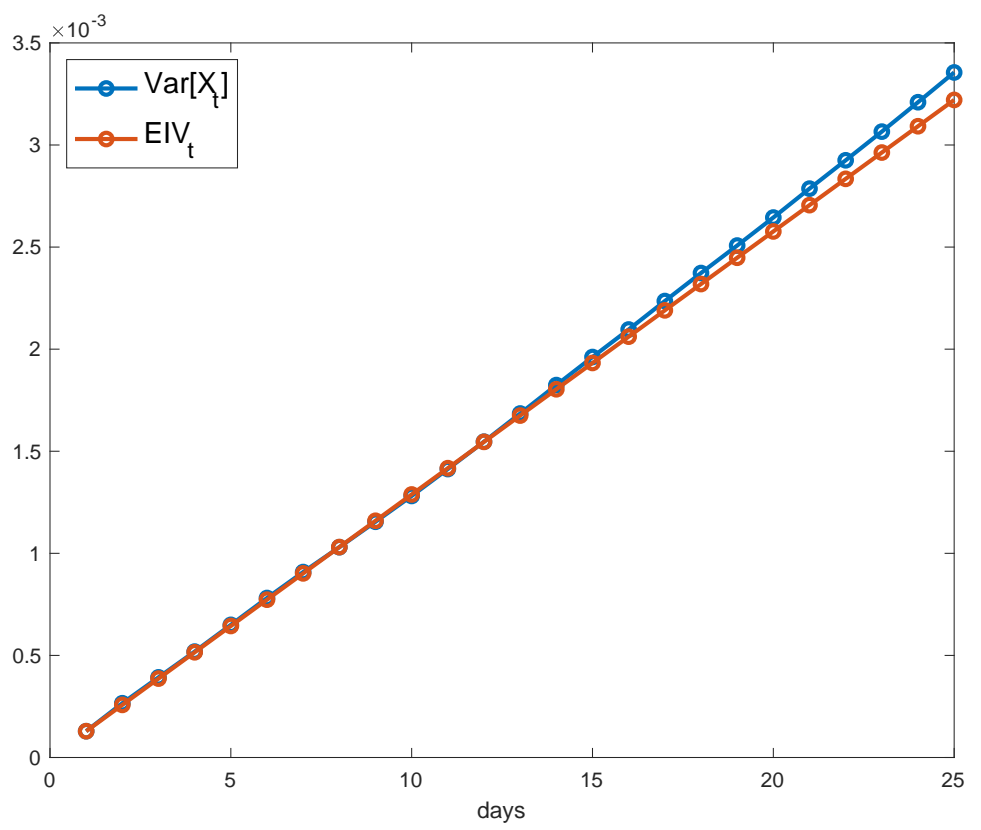

Figure 6: The interaction effect of the S\&P 500 over time

\section{Conclusion}

In this paper, we study the impact of the leverage effect on the return distribution. In particular, we focus on the marginal distribution since it can be directly formed from the empirical data. We find that the leverage effect is important, but it need not have a big impact on the return distribution. A strong leverage effect can be negated by a strong mean-reversion effect. Hence, when studying the return distribution properties, one must consider the interaction effect, which is the mixture of the leverage effect and the mean-reversion effect.

The interaction effect is not directly measurable. Our measurement relies on the dynamics of the marginal variance, which can be estimated accurately. A direct impact of our measurement is that the annualized marginal variance of the return will grow over time until it converges for a strong interaction effect, but will barely change for a weak interaction effect. The study of the interaction effect also shed light on the performance of the SRTR. Even with a weak mean- 
reversion effect, if the interaction effect is strong, the SRTR will not give an accurate approximation.

When applying our methodology to empirical data, we observed some interesting phenomena. We found that the interaction effect is stronger for small firms than large firms. We resolved the puzzle that fitting the S\&P 500 return distribution is insensitive to the leverage effect. By employing the GH distribution, a marginal distribution for a class of stochastic volatility model with no leverage effect, we confirmed that the S\&P 500 return distribution can be fitted well without the leverage effect. By our method, we found the interaction effect for $\mathrm{S} \& \mathrm{P} 500$ is weak, which we believe is the explanation to the puzzle.

\section{Acknowledgments}

We thank Robert Anderson and Lisa Goldberg for helpful discussions and comments.

\section{References}

Abate, J., Whitt, W., 1995. Numerical inversion of laplace transforms of probability distributions. ORSA Journal on computing 7, 36-43.

Ahn, D.H., Gao, B., 1999. A parametric nonlinear model of term structure dynamics. The Review of Financial Studies 12, 721-762.

Aït-Sahalia, Y., 2002. Maximum likelihood estimation of discretely sampled diffusions: a closed-form approximation approach. Econometrica 70, 223262.

Ait-Sahalia, Y., Fan, J., Li, Y., 2013. The leverage effect puzzle: Disentangling sources of bias at high frequency. Journal of Financial Economics 109, $224-$ 249.

Bakshi, G., Ju, N., Ou-Yang, H., 2006. Estimation of continuous-time models with an application to equity volatility dynamics. Journal of Financial Economics 82, 227-249. 
Bandi, F.M., Renò, R., 2012. Time-varying leverage effects. Journal of Econometrics 169, 94-113.

Barndorff-Nielsen, O., 1977. Exponentially decreasing distributions for the logarithm of particle size. Proceedings of the Royal Society of London. A. Mathematical and Physical Sciences 353, 401-419.

Barndorff-Nielsen, O., Halgreen, C., 1977. Infinite divisibility of the hyperbolic and generalized inverse gaussian distributions. Probability Theory and Related Fields 38, 309-311.

Bauwens, L., Laurent, S., Rombouts, J.V., 2006. Multivariate garch models: a survey. Journal of applied econometrics 21, 79-109.

BenSaïda, A., Slim, S., 2016. Highly flexible distributions to fit multiple frequency financial returns. Physica A: Statistical Mechanics and its Applications 442, 203-213.

Black, F., 1976. Studies of stock market volatility changes. 1976 Proceedings of the American Statistical Association Bisiness and Economic Statistics Section

Bonato, M., 2011. Robust estimation of skewness and kurtosis in distributions with infinite higher moments. Finance Research Letters 8, 77-87.

Campbell, J.Y., Hentschel, L., 1992. No news is good news: An asymmetric model of changing volatility in stock returns. Journal of financial Economics $31,281-318$.

Chen, D., Anderson, R., 2018. Predicting portfolio return volatility at medium horizons. Available at SSRN 3302940 .

Chicheportiche, R., Bouchaud, J.P., 2011. Goodness-of-fit tests with dependent observations. Journal of Statistical Mechanics: Theory and Experiment 2011, P09003. 
Chorro, C., Guegan, D., Ielpo, F., Lalaharison, H., 2018. Testing for leverage effects in the returns of us equities. Journal of Empirical Finance 48, 290-306.

Christie, A.A., 1982. The stochastic behavior of common stock variances: Value, leverage and interest rate effects. Journal of financial Economics 10, 407-432.

Cont, R., 2001. Empirical properties of asset returns: stylized facts and statistical issues .

Cox, J.C., Ingersoll Jr, J.E., Ross, S.A., 2005. A theory of the term structure of interest rates, in: Theory of Valuation. World Scientific, pp. 129-164.

Crow, E.L., Siddiqui, M., 1967. Robust estimation of location. Journal of the American Statistical Association 62, 353-389.

Danielsson, J., Zigrand, J.P., 2006. On time-scaling of risk and the square-rootof-time rule. Journal of Banking \& Finance 30, 2701-2713.

Drgulescu, A.A., Yakovenko, V.M., 2002. Probability distribution of returns in the heston model with stochastic volatility. Quantitative finance 2, 443-453.

Duan, J.C., 1995. The garch option pricing model. Mathematical finance 5, $13-32$.

Eberlein, E., Keller, U., et al., 1995. Hyperbolic distributions in finance. Bernoulli 1, 281-299.

Figlewski, S., Wang, X., 2000. Is the'leverage effect'a leverage effect? Available at SSRN 256109 .

Forde, M., Jacquier, A., 2009. Small-time asymptotics for implied volatility under the heston model. International Journal of Theoretical and Applied Finance 12, 861-876.

French, K.R., Schwert, G.W., Stambaugh, R.F., 1987. Expected stock returns and volatility. Journal of financial Economics 19, 3-29. 
Heston, S.L., 1993. A closed-form solution for options with stochastic volatility with applications to bond and currency options. The review of financial studies $6,327-343$.

Hinkley, D.V., 1975. On power transformations to symmetry. Biometrika 62, $101-111$.

Kim, T.H., White, H., 2004. On more robust estimation of skewness and kurtosis. Finance Research Letters 1, 56-73.

Lamoureux, C.G., Lastrapes, W.D., 1990. Heteroskedasticity in stock return data: Volume versus garch effects. The journal of finance 45, 221-229.

Nelson, D.B., 1991. Conditional heteroskedasticity in asset returns: A new approach. Econometrica: Journal of the Econometric Society , 347-370.

Peligrad, M., 1986. Recent advances in the central limit theorem and its weak invariance principle for mixing sequences of random variables (a survey), in: Dependence in probability and statistics. Springer, pp. 193-223.

Sepp, A., 2008. Pricing options on realized variance in the heston model with jumps in returns and volatility. Journal of Computational Finance 11, 33-70.

Seshadri, V., 2004. Halphen's laws. Encyclopedia of statistical sciences 5.

Silva, A.C., Yakovenko, V.M., 2003. Comparison between the probability distribution of returns in the heston model and empirical data for stock indexes. Physica A: Statistical Mechanics and its Applications 324, 303-310.

Sørensen, M., 1997. Exponential family inference for diffusion models .

Valsa, J., Brančik, L., 1998. Approximate formulae for numerical inversion of laplace transforms. International Journal of Numerical Modelling: Electronic Networks, Devices and Fields 11, 153-166.

Wang, C.D., Mykland, P.A., 2014. The estimation of leverage effect with highfrequency data. Journal of the American Statistical Association 109, 197-215. 
Wang, J.N., Yeh, J.H., Cheng, N.Y.P., 2011. How accurate is the square-rootof-time rule in scaling tail risk: A global study. Journal of Banking \& Finance $35,1158-1169$.

Weiss, M.S., 1978. Modification of the kolmogorov-smirnov statistic for use with correlated data. Journal of the American Statistical Association 73, 872-875.

Yu, J., 2005. On leverage in a stochastic volatility model. Journal of Econometrics 127, 165-178. 